\title{
Equivalência de Estímulos e Redução de Dificuldades na Solução de Problemas de Adição e Subtração ${ }^{1}$
}

\author{
Marcelo Henrique Oliveira Henklain ${ }^{2}$ \\ João dos Santos Carmo \\ Universidade Federal de São Carlos
}

\begin{abstract}
RESUMO - Foi avaliado se a formação de classes de equivalência entre quatro tipos de problemas de adição aumenta a porcentagem de acertos em problemas com diferentes formas de apresentação, posições da incógnita e estruturas semânticas. Participaram oito estudantes do $2^{\circ}$ ao $5^{\circ}$ ano do Ensino Fundamental, com baixa porcentagem de acertos na resolução de problemas de adição e subtração. Após a formação da classe de equivalência ocorreram mais acertos em todos os tipos de problemas. Investigou-se, então, o efeito do ensino de algoritmos sobre esse desempenho. Não foi observado um padrão homogêneo de melhora após esse ensino. No teste de generalização, os participantes não cometeram erros. Verificou-se que os procedimentos adotados contribuíram para a melhora da eficácia do comportamento de resolver problemas.
\end{abstract}

Palavras-chave: resolução de problemas, adição e subtração, equivalência de estímulos, ensino de algoritmos

\section{Stimulus Equivalence and Difficulties in Solving Addition and Subtraction Problems}

\begin{abstract}
This study investigated whether the formation of equivalence classes among four types of addition problems increases the percentage of correct responses in problems with different forms of presentation, unknown positions and semantic structures. Eight $2^{\text {nd }}$ to $5^{\text {th }}$ grade Elementary School students with low percentages of correct responses in solving addition and subtraction problems participated in this study. After the formation of equivalence classes, an increase of correct responses in all problem types was observed. Then, the effect of teaching algorithms on solving addition and subtraction problems was investigated. No homogeneous pattern of improvement was observed after teaching these algorithms. Participants committed no errors on the generalization test. The results indicate that the adopted procedures contributed to improve the effectiveness of problem solving behavior.
\end{abstract}

Key words: problem solving, addition and subtraction, stimulus equivalence, teaching of algorithms

Resolver problemas de adição e subtração é fundamental para o aprendizado de operações matemáticas mais complexas (Magina, Santana, Cazorla, \& Campos, 2010; Nunes \& Bryant, 1996). Infelizmente, existe grande dificuldade no ensino desse comportamento (Bryant, 2011; Costa, Galvão, $\&$ Ferreira, 2008; Sophian, 1996). Características como forma de apresentação, estrutura semântica dos problemas escritos e posição da incógnita $(a+b=c)$ têm sido destacadas como geradoras de dificuldades (Fayol, 1992; Fossa \& Sá, 2008; Haydu, Costa, \& Pullin, 2006; Nesher, Greeno, \& Riley, 1982; Verschaffel \& De Corte, 1997).

Em um estudo com 63 estudantes do $4^{\circ}$ ano do Ensino Fundamental, Resnick e Rosenthal (1974) demonstraram que a posição da incógnita afeta o comportamento de resolver

1 Apoio: Apoiado pela Fundação de Amparo à Pesquisa do Estado de São Paulo (FAPESP), processo 2011/16925-8, mediante a concessão de bolsa de mestrado, sendo que os dados desta pesquisa foram coletados durante a vigência da bolsa CAPES. As opiniões, hipóteses e conclusões ou recomendações expressas neste material são de responsabilidade dos autores e não necessariamente refletem a visão da FAPESP. Apoiado pelo MCT no âmbito do Edital 15/2008, com auxílio do CNPq (\#573972/2008-7) e da FAPESP (\#2008/57705-8).

2 Endereço para correspondência: Universidade Federal de São Carlos, Centro de Educação e Ciências Humanas, Rodovia Washington Luis, Km 235 SP-310, CEP 13565-905 - São Carlos, SP, Brasil. E-mail: marcelo_henklain@hotmail.com problemas. Quando a incógnita estava no início do problema, ocorriam mais erros e o participante demorava mais para alcançar a solução correta. Também foi demonstrado que a ordem cronológica de menção dos eventos do problema, ordem direta (começo, mudança e fim) e ordem reversa (fim, mudança e começo), determinavam o seu grau de dificuldade.

Em outro estudo clássico, Hiebert (1982) examinou o efeito da posição da incógnita sobre a representação do problema com objetos concretos (cubos) e a solução do mesmo. Participaram 47 crianças do $2^{\circ}$ ano do Ensino Fundamental, que foram expostas a 36 problemas aritméticos com estrutura semântica de transformação. Pequenos cubos estavam disponíveis e foi dito aos participantes que poderiam utilizá-los para ajudar na resolução dos problemas. Os resultados mostraram que, dos problemas cujas incógnitas estavam na posição $c$, tanto adição quanto subtração, $55 \%$ foram representados com cubos. Essa porcentagem caiu para $40 \%$ em problemas com incógnita na posição $b$, e para $18 \%$, na posição $a$, sendo que as porcentagens de acerto foram maiores nos problemas representados com os cubos. Portanto, a posição da incógnita determinou se o problema foi ou não representado com cubos, o que afetou a probabilidade de o participante resolvê-lo corretamente.

Contudo, outros estudos indicaram que, a depender da forma de apresentação do problema, o efeito da posição da incógnita pode variar, ocorrendo, por exemplo, mais acertos 
em problemas com incógnitas em $a$ ou $b$ do que em $c$, ou uma diferença menor de desempenho diante de cada incógnita (Haydu et al., 2001).

Pesquisas demonstraram também o efeito da estrutura semântica sobre o comportamento de resolver problemas. Magina et al. (2010) expuseram 1021 alunos do $2^{\circ}$ ao $5^{\circ}$ ano do Ensino Fundamental a 12 problemas escritos de adição e subtração, com estruturas semânticas de combinação, transformação e comparação, envolvendo situações familiares e pequenos números. Os dados mostraram que quanto maior a complexidade do problema, menor o percentual de acertos, sendo que os problemas mais complexos foram aqueles com estrutura de comparação e todas as situações em que não havia relação direta (congruência semântica) entre as palavras-chave do enunciado do problema e a operação que deveria ser realizada para resolvê-lo.

Nessa linha de investigação, a Análise do Comportamento tem apresentado propostas para mapear e contornar dificuldades na resolução de problemas (Neef, Nelles, Iwata, \& Page, 2003). Iégas (2003) criou o software Arit-Fácil para auxiliar estudantes do $2^{\circ}$ ano a resolver problemas aritméticos. Foi avaliado, no pré-teste, o desempenho na solução de problemas de adição e subtração nas formas escrita e de algarismos. Em seguida, foi investigado se o ensino de resolução de problemas na forma de balança poderia melhorar o desempenho em relação ao pré-teste. Nesse tipo de representação, o objetivo é equilibrar a balança, deixando a mesma quantidade de elementos nos seus dois pratos. No pré-teste, houve menor porcentagem de acertos nos problemas escritos com incógnitas nas posições $a$ e $b$. Após o treino com a balança, a porcentagem de acertos aumentou, principalmente diante de problemas escritos de adição com incógnita nas posições $a$ e $b$. Essa pesquisa indicou uma forma viável de aumentar a efetividade do comportamento de resolver problemas aritméticos.

Com base em resultados positivos do uso da balança, Haydu, Costa e Pullin (2006) investigaram se a formação de classes de equivalência entre três diferentes formas de apresentação de problemas (problemas escritos, operação com algarismos e balança) melhoraria o desempenho de resolução de problemas de adição. Participaram sete alunos do $2^{\circ}$ ano do Ensino Fundamental ainda não expostos ao ensino escolar formal de resolução de problemas aritméticos. Eles foram aprovados em um teste de leitura e apresentaram no pré-teste porcentagem de acerto inferior a 70\%. Antes da aplicação do pré-teste, foi explicado aos participantes, mostrando cada tipo de problema, que a tarefa deles era encontrar o valor da incógnita. No treino de discriminação condicional, problemas na forma de balança foram apresentados como modelo (A) e os estímulos-comparação foram problemas nas formas de algarismo (B) ou escrita (C). Em seguida, foi testada a emergência de simetria (relações B-A e C-A), de transitividade (B-C) e simetria da transitividade (C-B). Após essa fase, foi aplicado o pós-teste. Os resultados mostraram que seis dos sete participantes apresentaram aumento superior a $20 \%$ entre pré e pós-teste, sendo que, assim como no pré-teste, a porcentagem de acertos nos problemas escritos continuou menor do que a alcançada nas demais formas de apresentação.
Esse efeito positivo pode ser explicado pelo fato de que as funções adquiridas por um membro de uma classe de estímulos podem ser transferidas para os demais membros (Sidman \& Tailby, 1982). Assim, as estratégias de solução controladas por uma determinada forma de apresentação do problema passam a ocorrer diante de novas formas inseridas na classe (Haydu et al., 2006). Esse procedimento é aplicável a contextos reais, como a sala de aula, e "[...] pode ser uma maneira de o professor levar o aluno a aprender que o comportamento (estratégia de resolução) apresentado em uma situação pode ser usado em situações que são semelhantes" (Haydu et al., 2006, p. 51).

Justamente pelos resultados promissores e pela demanda social por pesquisa na área, é que se torna relevante ampliar o número de investigações sobre outras condições que não foram estudadas por Iégas (2003) e Haydu et al. (2006). Nesses dois trabalhos não existe uma avaliação acerca do efeito da estrutura semântica sobre o desempenho dos participantes e se os procedimentos sugeridos podem melhorá-lo diante de qualquer tipo de estrutura semântica. No procedimento de Haydu et al. também não foi avaliado se a formação de um conjunto de classes de adição pode ajudar com os problemas de subtração. Por fim, o procedimento de Haydu et al. envolveu interação pesquisador-participante. Fica a dúvida, portanto, se um procedimento com uso preponderante do computador produziria resultados similares.

Considerando esses aspectos, o objetivo principal da presente pesquisa foi investigar o efeito da formação de classes de estímulos equivalentes com quatro diferentes formas de apresentação de problemas de adição sobre o comportamento de resolver problemas de adição e subtração. Em função do caráter translacional desta pesquisa, foi também avaliado o efeito do ensino de algoritmos para resolução de problemas de adição e de subtração sobre o desempenho dos participantes, estratégia adicional para produzir um padrão de elevada porcentagem de acertos.

\section{Método}

\section{Participantes}

Oito crianças com idade entre 8 e 11 anos, sete do sexo masculino (JM, FS, MS, MB, PR, HC e VG) e uma do sexo feminino (PS) participaram deste estudo. Uma estava cursando o $5^{\circ}$ ano (HC), duas, o $4^{\circ}$ ano (PS e FS) e cinco, o $2^{\circ}$ ano (JM, PR, MS, MB e VG) do Ensino Fundamental. Sete estudavam numa mesma escola pública municipal da cidade de São Carlos-SP e HC estudava numa escola estadual da mesma cidade. Todas participavam de um Projeto de Extensão cujo objetivo é ensinar crianças com dificuldades em leitura/escrita a ler e escrever. As crianças foram autorizadas por seus pais a participar da pesquisa mediante assinatura do Termo de Consentimento Livre e Esclarecido. Foram observadas as determinações do Comitê de Ética em Pesquisa em Seres Humanos da Universidade Federal de São Carlos e da norma 196/96 da Comissão Nacional de Ética em Pesquisa (Parecer n ${ }^{\circ}$. 013/2012). 


\begin{tabular}{ccc} 
Balança (A) Algarismo (B) & Coleção (C) & Problema Escrito (D) \\
\hline & $2+?$ & César tinha ? aviões. Ganhou mais \\
dois e ficou com cinco ao todo.
\end{tabular}

Figura 1. Formas de apresentação de problemas com incógnitas nas posições a, b e c.

O critério para participar da pesquisa foi ser capaz de ler problemas escritos simples e apresentar baixo desempenho em um conjunto de provas delineadas para o presente estudo, cujas características serão descritas na seção "Procedimentos". A aplicação dessas tarefas antes da introdução dos procedimentos de ensino foi denominada pré-teste e após os procedimentos foi denominada pós-teste. A avaliação de leitura era feita com base na fase do programa de leitura/ escrita (desenvolvido pelos pesquisadores do projeto de extensão) em que a criança se encontrava; foram selecionadas apenas crianças que estavam na última fase do programa de ensino, cujo propósito é treinar a leitura e escrita de algumas dificuldades da língua portuguesa (por exemplo, sons e usos corretos da letra $\mathrm{x}$ ) a crianças que já conseguem ler. Ao longo da sessão de pré-teste, o experimentador também perguntava se a criança estava conseguindo compreender os problemas escritos. Todos foram capazes de identificar os nomes dos personagens presentes nos problemas escritos, bem como o que possuíam ou haviam ganhado ou perdido e o valor que precisava ser descoberto.

O desempenho foi considerado baixo se o participante apresentasse porcentagem de acerto inferior a $70 \%$. É preciso destacar que todos os participantes já haviam sido expostos na escola a procedimentos para resolução de problemas de adição e subtração com um e dois dígitos e incógnita na posição $c$. Eles foram selecionados para participar do estudo porque apresentaram baixa porcentagem de acertos nas provas do pré-teste.

\section{Local e Materiais}

A coleta foi realizada numa sala da biblioteca da Universidade Federal de São Carlos, onde funciona uma unidade do Projeto de Extensão que atende crianças com dificuldade em leitura/escrita. As sessões foram programadas no software ProgMTS (Marcicano, Carmo, \& Prado, 2011). Apenas nas sessões de pré e pós-teste, utilizou-se também o software Excel $^{\circledR} 2007$ para apresentar problemas escritos e na forma de balança. Essa mudança ocorreu porque esses estímulos ficaram pequenos na tela do ProgMTS, dificultando a visualização.

No pré-teste, pós-teste, sessões de treino e teste, os participantes foram expostos a quatro tipos diferentes de apresentação de problemas (ver Figura 1): a) problemas na forma de algarismo; b) problemas na forma de coleções de elementos (círculos e triângulos pretos); c) problemas escritos; e d) problemas na forma de balança. A prova com problemas na forma de balança continha problemas escritos, que funcionavam como instruções sobre o tipo de problema que estava sendo representado pela balança. O objetivo foi facilitar a compreensão dos participantes ao ser estabelecida uma relação de igualdade entre o problema escrito e o que era mostrado na balança. Também no intuito de facilitar a compreensão, os valores empregados nos problemas estavam na forma de algarismos e não na forma escrita, como era o caso na prova com problemas escritos. Um exemplo de instrução para problema de adição na forma de balança foi "O prato B têm 9 bolas verdes. Precisamos colocar ? bolas pretas e 6 bolas azuis no prato A para equilibrar a balança"; um exemplo de instrução para problema de subtração foi "O prato B têm 4 bolas verdes. O prato A têm ? bolas pretas. Precisamos retirar 5 bolas do prato A para equilibrar a balança". Para todas as formas de apresentação havia problemas com incógnita nas posições $a, b$ e $c$.

Apenas no pré-teste, pós-testes e no treino do algoritmo de subtração apareceram problemas de subtração. Portanto, ao longo das sessões de treino de discriminações condicionais, assim como na sessão de treino do algoritmo de adição, os problemas eram operações de adição cujo resultado estava entre 1 e 9. Foi feita variação de posição apenas dos estímulos-comparação, variação de forma e posição em relação aos problemas na forma de coleções e variação de ordem de apresentação dos problemas entre as sessões.

\section{Procedimento}

A Figura 2 exibe um diagrama com todas as fases do presente experimento.

Antes do pré-teste, os participantes assistiram a um vídeo que apresentava o problema escrito e mostrava como resolvê-lo, isto é, havia uma indicação explícita de que o problema deveria ser lido e o que valor a ser descoberto era $\mathrm{o}$ indicado pela interrogação com a cor vermelha. $\mathrm{O}$ intervalo entre tentativas, independente da sessão, era de 1 segundo. Em todos os treinos, respostas corretas produziam elogios verbais liberados pelo software e a apresentação de nova tentativa. Erros eram seguidos pela dica verbal "não, não é" e reapresentação da tentativa por até três vezes. Nas tentativas de teste não havia qualquer consequência diferencial. 


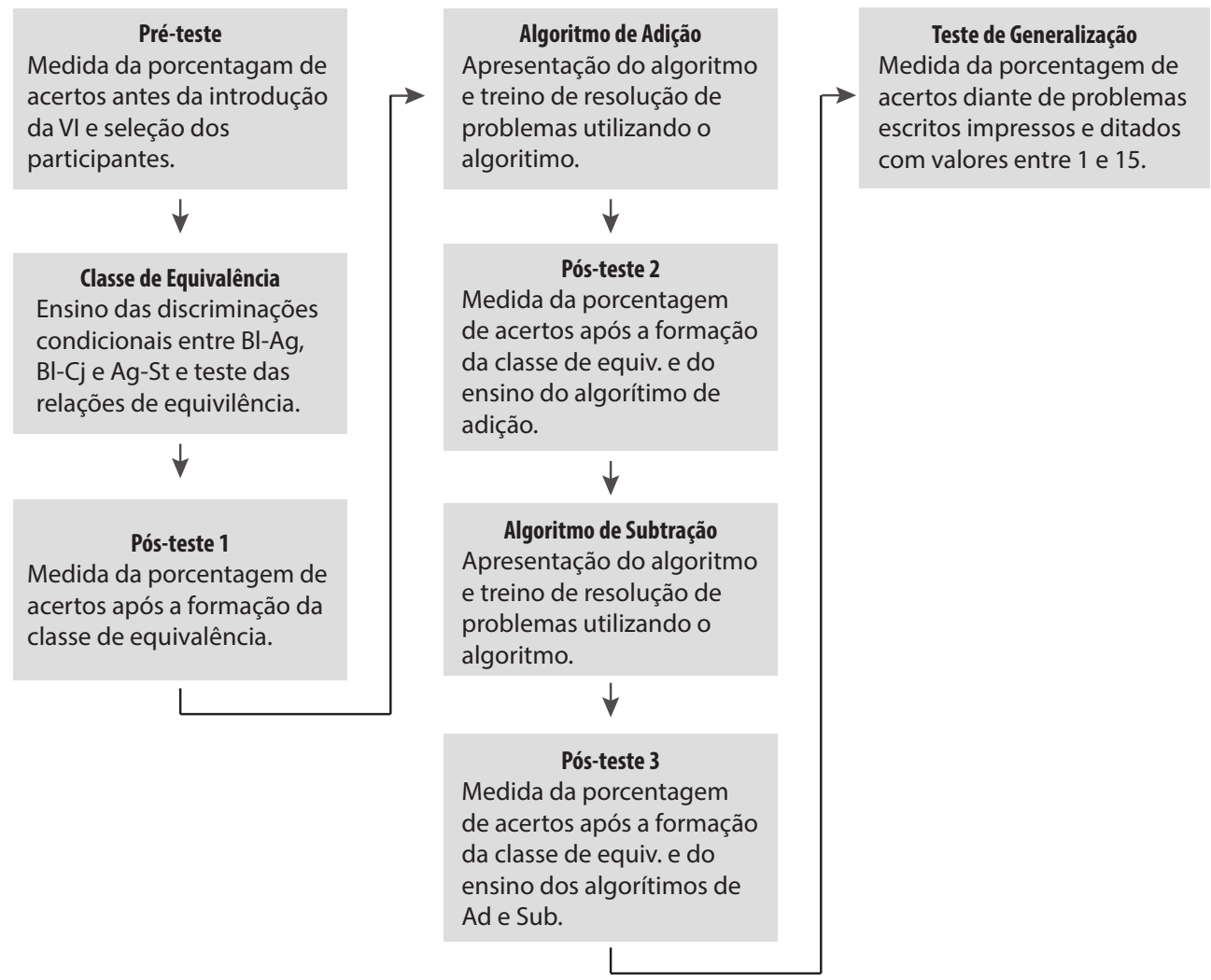

Figura 2. Fases da pesquisa a que os participantes foram expostos ( $\mathrm{Ad}=$ Adição; $\mathrm{Ag}=$ Forma de algarismo; $\mathrm{Bl}=$ Forma de balança; $\mathrm{Cl}=$ Forma de coleção; $\mathrm{St}=$ Problema escrito; $\mathrm{Sub}=$ Subtração).

Tentativas de teste em sessões de treino foram denominadas de sondas e apareciam de forma alternada ao longo da sessão. Nas sessões de instrução e treino, que envolviam resolução de problemas na forma de balança, a consequência para acertos era a apresentação de vídeo com uma balança sendo equilibrada e a consequência para erros era idêntica às das demais tentativas.

\section{Pré-teste e pós-testes}

No início de qualquer prova do pré-teste e do pós-teste, um problema-exemplo foi resolvido pelo experimentador junto com o participante para garantir que este saberia resolver as tarefas propostas. Nos problemas sob a forma de balança, antes de resolver o problema-exemplo o experimentador explicava o funcionamento da balança. Nas sessões de treino de resolução de problemas havia um problema no lugar do estímulo-modelo, nove estímulos-comparação (sendo que apenas um era a resposta correta) e o comando "Que número deve ser colocado no lugar desta interrogação para que esta conta fique correta?"; apresentar vários estímulos-comparação teve por objetivo diminuir as chances de que o participante pudesse acertar ao acaso.

Para cada forma de apresentação dos problemas foram construídas quatro provas. Cada prova era composta por seis problemas de matemática (todos sob uma mesma forma de apresentação), exceto a prova com problemas escritos. Desses seis problemas, três eram de adição e três de subtração. Havia dois problemas para cada posição da incógnita, um de adição e um de subtração; dois problemas com incógnita na posição $a$, dois na $b$ e dois na $c$.

A prova com problemas escritos continha 18 problemas, sendo nove de adição e nove de subtração. Para cada posição da incógnita havia três problemas de adição e três de subtração. Essa prova foi construída com mais questões porque foram empregados três diferentes tipos de estruturas semânticas: a) combinação, b) transformação, e c) comparação. Para cada estrutura semântica havia três questões: uma com incógnita na posição $a$, outra na $b$ e outra na $c$. Essas diferentes estruturas semânticas só foram utilizadas no contexto do pré-teste e dos pós-testes porque nas demais sessões foram utilizados apenas problemas escritos com estrutura de transformação.

A diferença básica entre os problemas de adição e os de subtração estava nos verbos ganhou ou perdeu, no caso da estrutura semântica de transformação; em expressões como "a mais que" ou "a menos que", no caso da estrutura semântica de comparação; e na ordem de apresentação das informações que sinalizava que o primeiro elemento deveria ser somado ao segundo para obter o resultado, ou que o segundo elemento apresentado deveria ser subtraído do primeiro, no caso da estrutura semântica de combinação. São fornecidos exemplos dos três tipos de estrutura semântica: a) combinação: "Numa sala há ? meninas e seis meninos. No total, são nove alunos"; b) transformação: "Pedrinho tinha ? carrinhos. Ganhou mais seis de presente e ficou com oito carrinhos ao todo"; c) comparação: "Mariana tinha ? figurinhas e Gabriela tinha três a mais que Mariana. Ao todo, Gabriela tinha sete figurinhas". 


\section{Formação de classes de equivalência}

Os treinos e testes de discriminações condicionais foram construídos no formato do procedimento de "escolha de acordo com o modelo" (matching to sample - MTS). No caso das sessões de teste, havia um estímulo-modelo, três estímulos comparação e o comando "Aponte o igual". Um dos estímulos-comparação correspondia ao estímulo-modelo (mesma ordem de apresentação dos valores e os mesmos valores). Nos demais estímulos-comparação, um continha a mesma posição da incógnita que o modelo (embora com valores diferentes nos demais termos da equação), e outro não tinha a mesma posição da incógnita, podendo ou não ter valores similares.

Antes do treino das discriminações condicionais, todos os participantes assistiram a um vídeo que explicava o funcionamento da balança e destacava que o objetivo era equilibrá-la. Foram empregados 12 tipos de problemas nas sessões de treino e de teste de equivalência, divididos em dois conjuntos de seis problemas cada (Conjunto 1 e Conjunto 2). No treino, o participante era exposto por três vezes a um conjunto de seis problemas e, se obtivesse porcentagem de acerto maior ou igual a $90 \%$, passava ao treino de um novo conjunto de seis problemas. Nas três vezes, o conjunto de problemas era o mesmo, mas foi feita variação na ordem de ocorrência de cada problema do conjunto. Para o ensino de uma relação foram programadas seis sessões, sendo três para o treino de cada conjunto de seis problemas. Nas tentativas de treino havia feedback para acerto e erro. Havia também 18 tentativas sem feedback (sondas para verificação de aprendizagem), atendendo ao critério de que para o aprendizado de uma determinada relação ser testado pela primeira vez era preciso o participante ter sido exposto a, pelo menos, duas tentativas de treino dessa relação. Se o participante apresentasse os índices de acerto esperados nos dois conjuntos de problemas $(90 \%)$ e nas sondas $(70 \%)$, passaria à fase seguinte que era o teste de equivalência. Caso contrário, repetiria as sessões do conjunto em que tivessem ocorrido mais erros.

Foram ensinadas as relações Balança (A) e Algarismo (B), Balança (A) e Coleção (C) e Algarismo (B) e Problema escrito (D), e testada a emergência das relações B-A, C-A, D-B, D-C, C-D, A-D, D-A, B-C e C-B. Nas fases de teste de equivalência, o critério de aprovação para a próxima fase de treino era apresentar pelo menos $70 \%$ de acerto. Caso não conseguisse, repetia-se o teste de equivalência na sessão subsequente. Se o desempenho continuasse baixo, eram reaplicadas as sessões de treino, com ênfase nos problemas que mais geraram dificuldades nos testes de equivalência. Ao final dessa fase foi aplicado o pós-teste 1 .

\section{Ensino de algoritmos}

Nas tentativas das sessões de instrução e treino havia um problema como estímulo-modelo, nove estímulos-comparação (sendo que apenas um era a resposta correta) e o comando "Que número deve ser colocado no lugar desta interrogação para que esta conta fique correta?". A diferença entre esse tipo de tentativa e a do pré-teste é que havia feedback para acerto e erro.
A fase de ensino do algoritmo para resolução de problemas de adição com incógnita nas posições $a$ e $b$ foi dividida em sessão de instrução (o experimentador descrevia como resolver o problema e resolvia alguns problemas durante a sessão) e treino (o participante, após a sessão de instrução, resolvia sozinho um conjunto de problemas). O algoritmo era apresentado em forma de instrução verbal, dada pelo experimentador, sobre como resolver o problema. A sessão de instrução foi composta por 24 problemas. O critério para seguir para a fase de treino era obter, pelo menos, $90 \%$ de acerto. A fase de treino foi composta por 48 problemas e o critério para seguir adiante era de, pelo menos, $90 \%$ de acerto. Nessas fases foram empregadas todas as formas de apresentação de problemas apresentadas na Figura 1, sendo que nos problemas escritos foi empregada apenas a estrutura de transformação.

Para problemas de adição com incógnita na posição $a$, foi dada a seguinte instrução: "Veja quanto você tem aqui no resultado. Agora veja que você tem a interrogação - não sabemos quanto ela vale - mais esse valor aqui. Bom, você não sabe quanto vale a interrogação, mas conhece esse valor que está sendo somado com ela. Se você já tem isso, basta contar quanto falta para chegar ao resultado. Conte nos dedos. Se você já tem isso aqui, vá contando até chegar ao resultado. Quantos dedos você levantou para chegar ao resultado? Esse é o valor da interrogação". Para problemas com incógnita na posição $b$, a instrução era semelhante, enfatizando-se que a interrogação mudou de lugar, mas o procedimento era o mesmo. Nos problemas com incógnita na posição $c$, foi enfatizado que o procedimento era somar os dois valores iniciais para chegar ao resultado. $\mathrm{O}$ experimentador avisou os participantes que eles poderiam resolver os problemas da forma que preferissem e forneceu alguns exemplos ao comentar que poderiam resolver os problemas usando os dedos, riscando traços num papel ou não utilizando qualquer representação externa. Ao final das instruções e treinos de resolução de problemas aplicou-se o pós-teste 2 .

A fase de ensino do algoritmo de resolução de problemas de subtração com incógnitas nas posições $a$ e $b$ foi dividida em sessão de instrução (ensino do algoritmo) e treino. A sessão de instrução foi composta por 19 problemas e o critério para seguir para a fase de treino foi obter pelo menos $90 \%$ de acerto. A fase de treino foi composta por 12 problemas, com o mesmo critério para avançar utilizado na fase anterior.

Para os problemas de subtração com incógnita na posição $a$ foi dada a instrução: "Veja que agora a conta é de menos. Você tem um número que não conhece - representado pela interrogação - menos este aqui, que sabemos qual é. O resultado é este. O que podemos fazer para descobrir o valor da interrogação? Se nós tiramos dessa quantidade aqui esta outra quantidade, e sobrou isto aqui de resultado, basta eu somar essa quantidade-resultado com essa quantidade que eu tirei da interrogação para descobrir quanto eu tinha inicialmente". A instrução para problemas de subtração com incógnita na posição $b$ foi: "Agora temos uma situação diferente. Sabemos o quanto temos aqui no começo, mas não sabemos - veja a interrogação - quanto foi tirado. Conhecemos também o resultado. O que fazer para encontrar a interrogação? Se eu tirei essa quantidade-interrogação e sobrou isso de resultado, basta fazer a continha de quanto eu tenho, menos o resulta- 
do, para saber quanto teria que ser retirado desse valor aqui para sobrar este resultado aqui". No ensino do algoritmo de subtração, o experimentador utilizou papel e lápis para escrever o problema e enfatizar cada um de seus elementos. Preferiu-se, na exposição, apresentar problemas com coleções para destacar quantidades retiradas ou adicionadas. Após a instrução e treino de resolução de problemas de subtração foi aplicado o pós-teste 3 .

\section{Teste de generalização}

Esse teste foi composto por seis problemas escritos de adição e seis de subtração, sendo seis com estrutura semântica de transformação na modalidade escrita (na tela do computador) e seis na modalidade ditada (lidos pelo experimentador). Em todos os casos, o participante deveria verbalizar a resposta e o experimentador registrava o acerto ou o erro. O objetivo desse teste foi avaliar se os participantes conseguiriam resolver problemas mais complexos, o que poderia indicar que os procedimentos de ensino adotados produziram ganhos de repertório não restritos apenas aos estímulos empregados nas sessões de treino e de teste.

\section{Resultados}

Os oito participantes foram expostos a todas as fases do experimento, mas nem todos no mesmo ano letivo e nem todos foram expostos à mesma quantidade de tentativas de discriminações condicionais. Esse procedimento foi adotado porque não haveria tempo para realizar todas as sessões antes do final do período letivo e não havia garantias de que a coleta poderia ser retomada no ano subsequente. Os participantes JM e VG passaram por uma fase reduzida de treino de discriminações condicionais. Na relação balança-coleção, JM foi exposto a 36 tentativas de treino do Conjunto 1 de problemas e 18 testes desse mesmo conjunto, e 12 tentativas de treino do Conjunto 2 de problemas e seis de testes desse mesmo conjunto; na relação algarismo-problema escrito, JM foi exposto, nos dois conjuntos, a 12 tentativas de treino e seis de teste. No caso de VG, houve alteração apenas no treino da relação algarismo-problema escrito, que foi composto por 12 tentativas de treino e seis de teste para os problemas dos Conjuntos 1 e 2 .

Ao final do ano letivo, a coleta foi finalizada integralmente apenas com HC, PS, MB e PR. Os participantes JM, FS, MS e VG não finalizaram todas as fases da pesquisa: JM e FS foram expostos a todas as fases até o pós-teste 1 e ao teste de generalização; MS foi exposto ao pós-teste 2 e ao teste de generalização; VG não finalizou todas as sessões de treino das discriminações condicionais, mesmo com a redução no número de tentativas. No ano seguinte, a pesquisa pôde ser retomada e a coleta foi finalizada com esses participantes.

\section{Desempenho no pré-teste}

A Tabela 1 exibe a porcentagem média de acertos por participante e a média de acertos de todos os participantes, considerando tipo de operação, forma de apresentação, estrutura semântica e posição da incógnita, no pré-teste e nos três pós-testes.

Embora com uma média geral de 54\% de acertos, os participantes apresentaram baixa porcentagem de acertos em alguns tipos de problemas. Essas baixas porcentagens envolveram problemas de subtração (47\% de acertos), quando comparados aos problemas de adição (61\% de acertos); em relação à forma de apresentação, os problemas que produziram menos acertos foram os problemas escritos (41\%) e a estrutura semântica com menor porcentagem de acertos foi a de comparação (com porcentagem média de acertos de $35 \%$ ). Nota-se também que os participantes acertaram menos problemas com incógnita na posição $a(47 \%$ de acertos) em relação aos problemas com incógnita em $b$ (54\% de acerto) e $c(60 \%$ de acerto $)$.

\section{Formação das classes}

$\mathrm{Na}$ análise da porcentagem de acertos dos participantes nas sondas e testes de equivalência, verificou-se que JM, MB, FS, MS, HC, PS, PR e VG apresentaram elevada porcentagem de acertos em praticamente todas as tentativas de testes/treinos; em todas as relações testadas, as porcentagens estavam acima de $77 \%$ de acertos, sendo que a média foi de $95 \%$ de acertos. Esse resultado sugere que os participantes formaram, ao longo da pesquisa, a classe de equivalência entre as diferentes formas de apresentação dos problemas. Apenas PR e VG precisaram de dois blocos adicionais de treino para alcançar os critérios de desempenho esperados.

\section{Desempenho nos pós-testes}

Conforme pode ser observado na Tabela 1 , há uma clara mudança no desempenho médio dos participantes, de $54 \%$ de acertos no pré-teste para $69 \%$ de acerto no pós-teste 1 . É interessante notar que, em todos os tipos de problema, há sempre mais acertos que erros, padrão não encontrado no pré-teste, no qual, em média, haviam mais erros que acertos nos problemas de subtração, nos problemas nas formas de balança e escrita, em todos os tipos de estrutura semântica e nos problemas com incógnita na posição $a$.

Após o primeiro pós-teste, quando foram ensinados os algoritmos, observou-se que o desempenho geral continuou crescendo (aumentando a porcentagem de acertos), mas com variações significativas entre os participantes. A partir dessa fase, apenas para que seja possível destacar os principais resultados, os participantes podem ser divididos em três grupos: aqueles que apresentaram aumento da porcentagem de acertos ao longo de todo o restante do experimento, aqueles que acertaram mais entre os pós-testes 1 e 2 , mas não entre os pós-testes 2 e 3 , e aqueles que diminuíram a porcentagem de acertos entre os pós-testes 1 e 2 e aumentaram entre os pós-testes 2 e 3 . Os participantes MS e VG representam o primeiro grupo. Ambos apresentaram melhoras ao longo de todo o experimento: 5,6\% (MS) e 34\% (VG) entre o pré-teste e o pós-teste $1 ; 11,1 \%$ (MS) e $22 \%$ (VG) entre o pós-teste 1 e o pós-teste 2 ; e 13,9\% (MS) e 19\% (VG) entre o pós-teste 2 e 
Tabela 1. Porcentagem média de acertos nas sessões de pré-teste e pós-testes 1,2 e 3.

\begin{tabular}{|c|c|c|c|c|c|c|c|c|c|c|c|c|c|c|}
\hline \multirow{2}{*}{ Part. } & \multirow{2}{*}{ Testes } & \multicolumn{2}{|c|}{ Operação } & \multicolumn{4}{|c|}{ Forma } & \multicolumn{3}{|c|}{ Estrutura } & \multicolumn{3}{|c|}{ Incógnita } & \multirow{2}{*}{ Média } \\
\hline & & Ad & Sub & Ag & Bl & $\mathrm{Cl}$ & St & TF & $\mathbf{C P}$ & CB & $\mathbf{a}$ & $\mathbf{b}$ & c & \\
\hline \multirow{4}{*}{$\begin{array}{l}\text { JM } \\
2^{\circ} \text { ano }\end{array}$} & Pré & 100 & 78 & 83 & 100 & 100 & 83 & 83 & 83 & 83 & 75 & 92 & 100 & 89 \\
\hline & Pós1 & 100 & 94 & 100 & 100 & 100 & 94 & 83 & 100 & 100 & 92 & 100 & 100 & 97 \\
\hline & Pós2 & 83 & 83 & 83 & 83 & 50 & 94 & 100 & 83 & 100 & 67 & 100 & 83 & 83 \\
\hline & Pós3 & 100 & 100 & 100 & 100 & 100 & 100 & 100 & 100 & 100 & 100 & 100 & 100 & 100 \\
\hline \multirow{4}{*}{$\begin{array}{l}\text { MS } \\
2^{\circ} \text { ano }\end{array}$} & Pré & 50 & 33 & 83 & 67 & 83 & 6 & 0 & 0 & 17 & 33 & 50 & 42 & 42 \\
\hline & Pós1 & 50 & 44 & 67 & 50 & 33 & 44 & 67 & 33 & 33 & 8 & 67 & 67 & 47 \\
\hline & Pós2 & 72 & 44 & 83 & 50 & 83 & 44 & 67 & 67 & 0 & 33 & 75 & 67 & 58 \\
\hline & Pós3 & 83 & 61 & 100 & 33 & 100 & 67 & 83 & 67 & 50 & 67 & 83 & 67 & 72 \\
\hline \multirow{4}{*}{$\begin{array}{l}\mathrm{MB} \\
2^{\circ} \text { ano }\end{array}$} & Pré & 44 & 33 & 83 & 0 & 50 & 33 & 50 & 17 & 33 & 25 & 33 & 58 & 39 \\
\hline & Pós1 & 78 & 78 & 100 & 50 & 67 & 83 & 83 & 67 & 100 & 83 & 92 & 58 & 78 \\
\hline & Pós2 & 100 & 72 & 100 & 67 & 83 & 89 & 100 & 83 & 83 & 75 & 92 & 92 & 86 \\
\hline & Pós3 & 78 & 67 & 100 & 50 & 83 & 67 & 83 & 33 & 83 & 83 & 58 & 75 & 72 \\
\hline \multirow{4}{*}{$\begin{array}{l}\text { PR } \\
2^{\circ} \text { ano }\end{array}$} & Pré & 44 & 28 & 33 & 17 & 50 & 39 & 83 & 17 & 17 & 25 & 33 & 50 & 36 \\
\hline & Pós1 & 72 & 50 & 67 & 67 & 83 & 50 & 50 & 33 & 67 & 58 & 58 & 67 & 61 \\
\hline & Pós2 & 50 & 17 & 67 & 17 & 17 & 33 & 17 & 33 & 50 & 25 & 17 & 58 & 33 \\
\hline & Pós3 & 89 & 94 & 83 & 100 & 83 & 94 & 100 & 100 & 83 & 92 & 92 & 92 & 92 \\
\hline \multirow{4}{*}{$\begin{array}{l}\text { VG } \\
2^{\circ} \text { ano }\end{array}$} & Pré & 28 & 17 & 50 & 17 & 67 & 0 & 0 & 0 & 0 & 17 & 25 & 25 & 22 \\
\hline & Pós1 & 61 & 50 & 83 & 50 & 100 & 33 & 33 & 33 & 33 & 58 & 42 & 67 & 56 \\
\hline & Pós2 & 78 & 78 & 100 & 83 & 100 & 61 & 83 & 17 & 83 & 83 & 75 & 75 & 78 \\
\hline & Pós3 & 100 & 94 & 100 & 100 & 100 & 94 & 100 & 83 & 100 & 92 & 100 & 100 & 97 \\
\hline \multirow{4}{*}{$\begin{array}{l}\text { FS } \\
4^{\circ} \text { ano }\end{array}$} & Pré & 78 & 56 & 83 & 83 & 67 & 56 & 50 & 67 & 50 & 42 & 75 & 83 & 67 \\
\hline & Pós1 & 83 & 67 & 100 & 50 & 67 & 78 & 67 & 67 & 100 & 58 & 83 & 83 & 75 \\
\hline & Pós2 & 78 & 67 & 83 & 50 & 100 & 67 & 83 & 67 & 50 & 67 & 67 & 83 & 72 \\
\hline & Pós3 & 89 & 94 & 100 & 100 & 100 & 83 & 83 & 100 & 67 & 92 & 83 & 100 & 92 \\
\hline \multirow{4}{*}{$\begin{array}{l}\text { PS } \\
4^{\circ} \text { ano }\end{array}$} & Pré & 56 & 50 & 100 & 33 & 83 & 33 & 33 & 33 & 33 & 67 & 42 & 50 & 53 \\
\hline & Pós1 & 61 & 50 & 100 & 50 & 83 & 33 & 33 & 33 & 33 & 67 & 42 & 58 & 56 \\
\hline & Pós2 & 78 & 72 & 100 & 50 & 83 & 72 & 83 & 67 & 67 & 67 & 83 & 75 & 75 \\
\hline & Pós3 & 78 & 67 & 100 & 50 & 83 & 67 & 83 & 50 & 67 & 75 & 67 & 75 & 72 \\
\hline \multirow{4}{*}{$\begin{array}{l}\mathrm{HC} \\
5^{\circ} \text { ano }\end{array}$} & Pré & 89 & 78 & 100 & 67 & 100 & 78 & 83 & 67 & 83 & 92 & 83 & 75 & 83 \\
\hline & Pós1 & 94 & 78 & 83 & 83 & 83 & 89 & 83 & 100 & 83 & 67 & 92 & 100 & 86 \\
\hline & Pós2 & 83 & 67 & 83 & 50 & 67 & 83 & 100 & 67 & 83 & 58 & 92 & 75 & 75 \\
\hline & Pós3 & 100 & 89 & 100 & 100 & 67 & 100 & 100 & 100 & 100 & 92 & 92 & 100 & 94 \\
\hline \multirow{2}{*}{ Média } & Pré & 61 & 47 & 77 & 48 & 75 & 41 & 48 & 35 & 40 & 47 & 54 & 60 & 54 \\
\hline & Pós & 81 & 70 & 91 & 66 & 80 & 72 & 78 & 66 & 72 & 69 & 77 & 80 & 75 \\
\hline
\end{tabular}

$\mathrm{Ad}=$ Adição; $\mathrm{Ag}=$ Algarismo $\mathrm{Bl}=$ Balança $; \mathrm{CB}=$ Estrutura de combinação; $\mathrm{CP}=$ Estrutura de comparação; $\mathrm{Cl}=\mathrm{Coleção} ; \mathrm{St}=\mathrm{Problema}$ escrito; $\mathrm{Sub}=$ Subtração; TF = Estrutura de transformação; $a$, b e c representam as posições da incógnita $(\mathrm{a}+\mathrm{b}=\mathrm{c}$ ou $\mathrm{a}-\mathrm{b}=\mathrm{c})$; Média $=\mathrm{Média}$ geral do participante no préteste e nos pós-testes.

o pós-teste 3. Os participantes JM, FS, HC e PR, por seu turno, melhoraram 8,3\% (JM e FS), 2,8\% (HC) e 25\% (PR) entre o pré-teste e o pós-teste 1. Entre o pós-teste 1 e o pós-teste 2, eles apresentaram queda na porcentagem de acertos $(13,9 \%$, $2,8 \%, 11,1 \%$ e $27,8 \%$, respectivamente). Na comparação entre o pós-teste 2 e o pós-teste 3 , esses quatro participantes melhoraram: 16,7\% (JM), 19,5\% (FS), 19,4\% (HC) e 58,3\%
(PR). Alguns participantes não alcançaram crescimento na porcentagem de acertos apenas entre os pós-testes 2 e 3 . Os participantes MB e PS apresentaram melhora entre o pré-teste e o pós-teste 1 (38,9\% e $2,8 \%$, respectivamente) e entre os pós-testes 1 e 2 ( $8,3 \%$ e $19,4 \%$, respectivamente), mas apresentaram pequena queda na fase final da pesquisa, entre os pós-testes 2 e 3: MB apresentou queda de 13,9\% e PS 2,8\%. 
Esses resultados sugerem que as sessões de ensino dos algoritmos interferiram no desempenho dos participantes, mas sem produzir um desempenho homogêneo; alguns participantes apresentaram aumento da porcentagem de acertos após o ensino do algoritmo de adição, outros apresentaram diminuição, e o mesmo ocorreu após o ensino do algoritmo de subtração. Houve regularidade apenas nos efeitos do procedimento de formação de classes de equivalência; sempre houve melhora após este procedimento, ainda que pequena (o ganho médio, em porcentagem de acertos, após esse procedimento, foi de $15,7 \%$ ).

A despeito das variações de desempenho a partir das sessões de ensino de algoritmos, é preciso destacar que nos pré-testes de PS, MS, MB, PR, VG há uma pequena porcentagem de acertos (53\%, 42\%, 39\%, 36\% e 22\% respectivamente) e, ao final do programa de ensino, considerando, por exemplo, apenas o último pós-teste, houve melhora na porcentagem de acertos desses quatro participantes: $72 \%, 72 \%, 72 \%, 92 \%$ e $97 \%$, respectivamente. Os participantes JM, FS e HC, por seu turno, apresentaram elevada porcentagem de acertos já no pré-teste, $89 \%, 67 \%$ e $83 \%$, respectivamente. Ao final do experimento, eles apresentaram, respectivamente, $100 \%$, $92 \%$ e $94 \%$ de acertos. No caso desses participantes, quando se analisa apenas a porcentagem de acertos inicial e final, é difícil afirmar categoricamente que a melhora de desempenho ocorreu em função dos procedimentos de ensino adotados. Mas, ao considerar todos os resultados da pesquisa, inclusive as quedas no desempenho, pode-se sugerir que o que foi ensinado nas sessões de fato interferiu no comportamento de resolver problemas.

Segundo o teste de Wilcoxon bicaudal, houve diferença estatisticamente significativa $(\mathrm{p}<0,05)$ entre o pré-teste e o pós-teste $1(z=-2,527 ; p=0,012)$ e os pós-testes 2 e $3(z=$ $-2,043 ; p=0,041)$. Também foram encontradas diferenças significativas entre o total de acertos em problemas de adição, entre o pré-teste e o pós-teste $1(z=-2,232 ; p=0,026)$; em problemas de subtração, entre o pré-teste e o pós-teste 1 $(z=-2,207 ; p=0,027)$ e entre os pós-testes 2 e $3(z=-2,113$; $p=0,035)$; em problemas na forma de algarismo, entre os pós-testes 2 e $3(z=-2,236 ; p=0,025)$, e na forma escrita, entre o pré-teste e o pós-teste $1(z=-2,384 ; p=0,017)$; em problemas com estrutura de comparação, entre o pré-teste e o pós-teste $1(z=-2,232 ; p=0,026)$, e de combinação, entre o pré-teste e o pós-teste $1(z=-2,214 ; p=0,027)$ e entre os pós-testes 2 e $3(z=-2,06$ e $p=0,039)$; em problemas com incógnita em $a$, entre os pós-testes 2 e 3 ( $z=-2,546$ e $p=$ $0,011)$, em $b$, entre o pré-teste e o pós-teste $1(z=-2,388$; $p=0,017)$, e em $c$, entre o pré-teste e o pós-teste $1(z=$ $-2,032 ; p=0,042$ ).

As melhoras de desempenho encontradas e os resultados do teste estatístico demonstram que o programa de ensino produziu aumento na porcentagem de acertos, inclusive, em problemas de subtração, problemas escritos, problemas com estrutura semântica de comparação e problemas com incógnita nas posições iniciais $(a \mathrm{e} b)$, todos eles problemas que produziam baixas porcentagens de acertos no início do experimento. De fato, pode-se notar, na Tabela 1, a partir do cálculo da média de desempenho de todos os participantes (porção inferior da tabela), essa melhora diante de todos os tipos de variáveis manipuladas na presente pesquisa. No teste de generalização, todos os participantes alcançaram $100 \%$ de acerto.

\section{Discussão}

Os resultados do presente estudo indicam que a formação de classes de equivalência com quatro diferentes formas de apresentação de problemas de adição melhorou o desempenho de alunos do $2^{\circ}$ ao $5^{\circ}$ ano do Ensino Fundamental na resolução de problemas de adição e subtração. Esse resultado foi observado mesmo diante de problemas que, inicialmente, conforme demonstrado pelo pré-teste, produziram dificuldades.

Observou-se, no pré-teste, que seis dos oito participantes apresentaram desempenho abaixo de $70 \%$ de acertos e apenas dois apresentaram desempenho entre $70 \%$ e $90 \%$ de acertos. Nessa fase, a maior parte dos erros cometidos aconteceu em problemas com incógnita na posição $a$, e a menor, em problemas com incógnita na posição $c$. Esse resultado confirma o que está descrito na literatura. Hiebert (1982) salienta que em problemas de adição e subtração com incógnita na posição $a$, seus participantes erraram mais e apresentaram maior dificuldade para modelar o problema que nos problemas com incógnita nas posições $b$ e $c$. Resnick e Rosenthal (1974) também identificaram mais erros em problemas com incógnita na posição $a$ do que na posição $c$, independentemente dos verbos empregados (ganhar ou perder) e da ordem cronológica de apresentação dos dados do problema. Fossa e Sá (2008), em sua revisão de literatura sobre resolução de problemas, também concluíram que a posição $a$ é a mais difícil, embora essa dificuldade diminua em função da idade (provável efeito da escolarização). A dificuldade com a posição da incógnita em $a$ e $b$ se deve ao fato de que uma nova habilidade deve ser aprendida (Haydu et al., 2006; Hiebert, 1982;); o procedimento para encontrar o resultado da soma " $2+2$ " não é o mesmo requisitado para encontrar o valor da posição $a$ na conta "?+2=4". Bryant (2011) explica que a criança precisa desenvolver a noção de inversão, o que significa aprender que as operações de adição e subtração são complementares. Assim, " $2+2=4 " \mathrm{e}$ " $4-2=2 "$ ".

Além da posição da incógnita, os problemas de subtração e os problemas escritos, especialmente aqueles com estruturas de comparação e combinação, geraram dificuldades. Esse resultado também é o esperado segundo a literatura (Fossa \& Sá, 2008). Surpreendeu o resultado de que cinco participantes erraram mais os problemas na forma de balança. Ele se contrapõe àqueles obtidos nos estudos de Iégas (2003) e Haydu et al. (2006), nos quais os problemas na forma de balança contribuíram para o aumento da porcentagem de acertos e a maior parte dos erros concentrou-se nos problemas escritos. Isso pode sinalizar a importância de um treino mais amplo para o ensino de como devem ser resolvidos problemas na forma de balança. É possível que as instruções fornecidas não tenham sido suficientes para que os participantes entendessem o seu funcionamento. Também é bastante provável que a presença de problemas escritos tenha aumentado a dificuldade dos problemas na forma de balança. Estudos futuros devem utilizar balanças sem a apresentação concomitante de problemas escritos. 
Ao final do primeiro pós-teste, todos os participantes apresentaram aumento da porcentagem de acertos em relação ao pré-teste. Este dado confirma os resultados de Haydu et al. (2006). Após o ensino do algoritmo de adição, quatro participantes (VG, MB, MS e PS) apresentaram aumento da porcentagem de acertos em relação ao pré-teste e ao pós-teste 1, três (PR, HC e JM) apresentaram diminuição da porcentagem de acertos em relação a esses dois testes e um (FS) apresentou diminuição de acertos apenas em relação ao seu desempenho no pós-teste 1. Após o ensino do algoritmo de subtração, seis (VG, PR, MS, FS, HC e JM) melhoraram o desempenho em relação aos testes anteriores, um (MB) apresentou diminuição da porcentagem de acertos em relação aos pós-testes anteriores, mas ficou acima do desempenho apresentado no pré-teste, e um (PS) apresentou desempenho menor apenas em relação ao pós-teste 2 . Não houve, portanto, nenhum participante que tenha terminado o estudo com desempenho abaixo do que havia apresentado no pré-teste.

É provável que o procedimento de ensinar primeiro o algoritmo de adição e depois o de subtração, numa outra sessão, possa ter levado os participantes a empregar o algoritmo de adição para resolver tanto os problemas de adição quanto de subtração. Essa hipótese pode explicar a variação de desempenho dos participantes observada no pós-teste $2 \mathrm{e}$ a recuperação de desempenho no pós-teste 3, após o ensino do algoritmo de subtração. Outra variável a ser considerada é que o treino realizado pode não ter sido suficiente para garantir que o desempenho dos participantes ficasse sob controle dos algoritmos. Portanto, regras erradas podem ter sido empregadas, o que explicaria os erros. Pode ter ocorrido também confusão entre os algoritmos apresentados pelo experimentador e aqueles ensinados na escola, ou seja, diante dos problemas a serem resolvidos, não houve controle eficaz do comportamento de solução, nem pelos algoritmos do experimentador, tampouco pelos da escola.

Sem os dados dos três participantes que apresentaram elevado índice de acerto no pré-teste (JM, FS e HC), verificou-se que os demais participantes (PS, MS, MB, PR e VG) apresentaram uma diferença média de acertos entre pré e pós-testes de $30 \%$. Considerando todos os participantes, a diferença média foi de $21 \%$. Essas médias de acertos se assemelham aos dados de Haydu et al. (2006), que documentaram aumento superior a $20 \%$ nas porcentagens médias de acertos em relação ao seu pré-teste. Esses resultados demonstram o crescimento da porcentagem de acertos em decorrência dos procedimentos de ensino adotados, interpretação que é fortalecida com base nos resultados do teste de Wilcoxon já apresentados.

No teste de generalização, os participantes acertaram todos os problemas, o que sugere que o comportamento de resolução de problemas não ficou sob controle restrito dos estímulos empregados nas sessões de treino.

\section{Considerações Finais}

O objetivo principal desta pesquisa foi investigar o efeito da formação de classes de estímulos equivalentes com quatro diferentes formas de apresentação de problemas de adição sobre o comportamento de resolver problemas de adição e subtração. Foi avaliado também o efeito do ensino de al- goritmos sobre o desempenho dos participantes, estratégia adicional para produzir um padrão de elevada porcentagem de acertos.

Os resultados, incluindo aqueles no teste de Wilcoxon, demonstraram a eficácia dos procedimentos de ensino adotados, em especial a formação da classe que gerou uma melhora de desempenho para todos os participantes. O ensino de algoritmo, por outro lado, gerou resultados positivos para alguns participantes, mas não para todos. Houve uma clara variação no desempenho, o que aponta para a necessidade de aprimorar as instruções para garantir desempenhos mais estáveis.

Esta pesquisa insere-se nos estudos que propõem e avaliam procedimentos que facilitam a resolução de problemas aritméticos. Os resultados obtidos corroboraram os de Haydu et al. (2006) e avançam ao demonstrar que: a) a formação apenas da classe de equivalência de problemas de adição pode produzir melhora no desempenho não só em problemas de adição, como também de subtração, e 2) que não só a estrutura semântica (transformação) utilizada no treino foi afetada positivamente, mas também as estruturas de comparação e combinação. Os resultados sugeriram também que o ensino de algoritmos pode ajudar a aumentar a porcentagem de acertos, mas é preciso garantir que o aluno não utilize algoritmos de adição em problemas de subtração, e vice-versa, e que não fique insensível ao aprendizado de novas formas de resolver um problema ou novos tipos de problema para os quais o algoritmo ensinado pode não ser adequado.

\section{Referências}

Bryant, P. (2011). Children`s understanding and use of inversion in arithmetic. In Comité Interamericano de Educación Matemática (Ed.), Anais da XIII Conferencia Interamericana de Educación Matemática (pp. 1-7), Recife: CIAEM.

Costa, A. L. M., Galvão, O. F., \& Ferreira, B. P. (2008). ARIT um software baseado em equivalência de estímulos dirigido a crianças com histórico de fracasso na aprendizagem de conceitos aritméticos. In: Sociedade Brasileira de Computação (Ed.), Anais do XIX Simpósio Brasileiro de Informática na Educação [CD], (pp. 125-134) Fortaleza: SBC.

Fayol, M. (1992). From number to numbers in use: Solving arithmetic problems. In J. Bideaud, C. Meljac, \& J. P. Fischer (Eds.), Pathways to number: Children's developing numerical abilities (pp. 283-306). New Jersey: Lawrence Erlbaum Associates.

Fossa, J. A., \& Sá, P. F. (2008). Uma distinção entre problemas aritméticos e algébricos. Revista Educação em Questão, 33(19), 253-278.

Haydu, V. B., Paranzini, A. C. S., Isquierdo, G. R., Ausec, H. O., Mazzo, I. M. B., Pires, I. T. M., ... \& Pimentel, N. S. (2001). Dificuldades e facilidades produzidas pela forma de apresentação de problemas aritméticos com a incógnita em diferentes posições. In M. C. Marquezine, M. A. Almeida \& E. D. O. Tanaka (Eds.), Perspectivas multidisciplinares em Educação Especial II (pp. 593-601). Londrina, PR: Eduel.

Haydu, V. B., Costa, L. P., \& Pullin, E. M. M. P. (2006). Resolução de problemas aritméticos: efeito de relações de equivalência entre três diferentes formas de apresentação dos problemas. Psicologia: Reflexão \& Crítica, 19, 44-52. 
Hiebert, J. (1982). The position of the unknown set and children's solutions of verbal arithmetic. Journal for Research in Mathematics Education, 13, 341-349.

Iégas, A. L. F. (2003). Software para a resolução de problemas aritméticos: o modelo da balança. (Dissertação de Mestrado não publicada). Universidade Estadual de Londrina, Paraná.

Magina, S. P. M., Santana, E. R. S., Cazorla, I. M., \& Campos, T. M. M. (2010). As estratégias de resolução de problemas das estruturas aditivas nas quatro primeiras séries do Ensino Fundamental. Zetetiké, 18(34), 15-50.

Marcicano, D. C., Carmo, J. S., \& Prado, P. S. T. (2011). Software ProgMTS: possibilidades de delineamento e condução de programas de ensino em Análise Experimental do Comportamento [Computer software ]. Anais da $41^{a}$ Reunião Anual da Sociedade Brasileira de Psicologia: formação e produção do conhecimento em psicologia, Belém.

Neef, N. A., Nelles, D. E., Iwata, B. A., \& Page, T. J. (2003). Analysis of precurrent skills in solving mathematics story problems. Journal of Applied Behavior Analysis, 36, 21-33.

Nesher, P., Greeno, J. G., \& Riley, M. S. (1982). The development of semantic categories for addition and subtraction. Educational Studies in Mathematics, 13(4), 373-394.

Nunes, T., \& Bryant, P. (1996). Giving meaning to addition and subtraction. In T. Nunes, \& P. Bryant (Eds.), Children doing mathematics (pp. 114-141). Oxford: Blackwell.
Resnick, L. B., \& Rosenthal, D. J. A. (1974). Children's solution processes in arithmetic word problems. Journal of Educational Psychology, 66, 817-825.

Sidman, M., \& Tailby, W. (1982). Conditional discrimination vs. matching to sample: An expansion of the testing paradigm. Journal of the Experimental Analysis of Behavior, 37(1), 5-22.

Sophian, C. (1996). The sum of the parts. In C. Sophian (Ed.), Children's numbers (pp. 73-88). Colorado: Westview Press.

Verschaffel, L., \& De Corte, E. (1997). Word problems: A vehicle for promoting authentic mathematical understanding and problem solving in the primary school? In T. Nunes, \& P. Bryant (Eds.), Learning and teaching mathematics: An international perspective (pp. 69-97). Hove, England: Psychology Press.

Recebido em 22.11.2012

Primeira decisão editorial em 01.02.2013

Versão final em 20.05.2013

Aceito em 20.05.2013 